\title{
Commentary: Beyond "TRIM" Benefits of $\beta$-Glucan by Blood Glucose and Lipid Balancing Potentials in Its Defense Against COVID-19
}

\author{
Nobunao Ikewaki ${ }^{1,2}$, Vidyasagar Devaprasad Dedeepiya ${ }^{3}$, Masaru Iwasaki ${ }^{4}$ \\ and Samuel J. K. Abraham ${ }^{3,4,5,6 *}$ \\ ${ }^{1}$ Department of Medical Life Science, Kyushu University of Health and Welfare, Nobeoka, Japan, ${ }^{2}$ Institute of Immunology, \\ Junsei Educational Institute, Nobeoka, Japan, ${ }^{3}$ The Mary-Yoshio Translational Hexagon (MYTH), Nichi-In Centre for \\ Regenerative Medicine (NCRM), Chennai, India, ${ }^{4}$ School of Medicine, University of Yamanashi, Chuo, Japan, ${ }^{5}$ Edogawa \\ Evolutionary Laboratory of Science (EELS), Edogawa Hospital, Tokyo, Japan, ${ }^{6}$ Life-systems through Immunology, Phylogeny \\ and Evolution (LIPE) Division, GN Corporation Co. Ltd., Kofu, Japan
}

Keywords: COVID-19, trained immunity, $\beta$-glucan, blood glucose, fasting plasma glucose, lipid, coagulopathy, TRIM

OPEN ACCESS

Edited by:

Vasso Apostolopoulos,

Victoria University, Australia

Reviewed by:

Boris Novakovic,

Royal Children's Hospital, Australia

${ }^{*}$ Correspondence:

Samuel J. K. Abraham

drsam@nichimail.jp;

drspp@nichimail.jp

Specialty section:

This article was submitted to

Vaccines and Molecular

Therapeutics,

a section of the journal

Frontiers in Immunology

Received: 27 November 2020 Accepted: 15 March 2021

Published: 29 March 2021

Citation:

Ikewaki N, Dedeepiya VD, Iwasaki M and Abraham SJK (2021) Commentary: Beyond "TRIM" Benefits of $\beta$-Glucan by Blood Glucose and Lipid Balancing Potentials in Its Defense Against COVID-19.

Front. Immunol. 12:620658. doi: 10.3389/fimmu.2021.620658
A Commentary on Could the Induction of Trained Immunity by $\beta$-Glucan Serve as a Defense Against COVID-19? By Geller A and Yan J. Front. Immunol. 2020 11:1782. doi: 10.3389/fimmu.2020.01782

\section{INTRODUCTION}

The 'hypothesis and theory' article by Geller and Yan (1) is an eye-opener to the innate memory based Trained Immunity (TRIM) response offered by $\beta$-glucans in a prophylactic setting for immune-modulation to abrogate the symptoms in COVID-19, preventing morbidity and death.

Their review (1) begins with the evolutionary background of TRIM and moves on to its first recognition by the scientific community based on the non-specific immune response induced by the Bacille Calmette-Guérin (BCG) vaccine administered for Mycobacterium tuberculosis (TB). The multi-faceted TRIM offered by $\beta$-Glucans by virtue of their antiviral properties through production of IL- $1 \beta$, TNF- $\alpha$, and IFN- $\gamma$, induction of "central" memory by enabling production of a repertoire of innate cells from hematopoietic stem cells (HSC) in the bone marrow which migrate to peripheral tissues to generate peripheral memory and the epigenetic regulation through pathways of Dectin-1 activation (1) are elaborately narrated.

\section{SIGNIFICANCE OF METABOLIC REGULATION OF TRIM}

Geller and Yan (1) primarily focus on the anti-viral response of the metabolic regulation wherein modulation of the transcription of immunogenic genes occur by metabolic switch from oxidative phosphorylation toward aerobic glycolysis mediated through the AKT/mTOR/HIF1 $\alpha$ pathway. Besides, it inhibits the LPS mediated upregulation of immune-responsive gene-1 (IRG-1) needed for itaconate generation. As high levels of itaconate and its derivatives inhibit key Type-I interferon production during viral infection, itaconate generation inhibited by this metabolic upregulation of 
$\beta$-glucans leads to increased Type I IFN response mediating antiviral response against SARS-CoV-2 (1).

\section{BEYOND "TRIM" BENEFITS OF $\beta$-GLUCANS}

Apart from the metabolic based anti-viral response explained by Geller and Yan (1) or the immune-modulation described by Rao et al. (2) in the defense against COVID-19, we would like to emphasize that regulation of blood glucose and lipid levels by $\beta$ Glucans as an indispensable tool of defense (3).

Diabetes, dyslipidemia and the immune system are inseparable and inter-connected factors of worsening the crisis post-COVID-19 infection. While high blood glucose levels lead to constant glucose recognition by $\mathrm{C}$ type lectin receptors which in turn lead to increased rate of inflammatory processes worsening the disease severity in COVID-19 (4), persistent chronic inflammation, decreased function of vascular endothelial cells and decreased immune function occurring in COVID-19 may actually underlie the pathogenesis of diabetes (5). Further, blood sugar levels increase the levels of clotting factors, causing endothelial dysfunction, enhanced platelet aggregation and activation, favoring a hypercoagulable pro-thrombotic state increase the risk of coagulopathy due to COVID-19 (6). High levels of low-density lipoprotein (LDL) lead to an increase in inflammatory gene expression and inflammasome activation through interaction with macrophages in atherosclerotic plaques causing worsening of the cytokine storm in COVID-19 (6). Low levels of high-density lipoprotein (HDL) are involved in the regulation of innate immune response and negatively regulates $\mathrm{T}$-cell activation and the expression of inflammatory mediators in macrophages and dendritic cells (7). Dyslipidemia also causes endothelial dysfunction. With endothelial dysfunction induced coagulopathy having been identified to be the major cause of organ damage and death in COVID-19 patients (7), the contribution by high blood glucose and lipid levels cannot be ignored while planning prophylactic and therapeutic strategies to combat COVID-19 (8).

\section{$\beta$-GLUCANS IN NORMALIZATION OF GLUCOSE AND LIPID LEVELS IN CLINICAL STUDIES}

$\beta$-glucans such as the AFO-202 black yeast derived $\beta$-glucan have been shown to maintain the fasting plasma glucose (FPG) levels and bring down the high levels in diabetic patients to normal values in human clinical studies $(9,10)$ which has been hypothesized to be beneficial in the defense against COVID-19 (11). It is noteworthy that high FPG levels at admission has been proven as a strong independent predictor of poor outcomes in COVID-19 in several studies $(12,13)$. Further, the AFO-202 $\beta$-glucan has been shown to decrease LDL levels, very low-density lipoprotein (VLDL) levels and triglycerides to the normal range and increase lower HDL levels to reach their normal levels in human clinical studies (14).

\section{MECHANISMS OF ADDITIONAL BENEFITS OFFERED BY $\beta$-GLUCANS IN TRIM AND METABOLISM}

Immuno-metabolic circuits are critical in TRIM (15). Induction of glycolysis leads to immunotolerant monocytes as seen in sepsis by their effects on posttranslational modification of effector molecules such as glyceraldehyde 3-phosphate dehydrogenase (GAPDH) (16). Insulin resistance that occurs in obesity and diabetes facilitate TRIM in macrophages to acquire a state that has reduced responses to pathogens through metabolic and epigenetic changes. Insulin sensitization to reverse this state has been hypothesized to be utilized for inducing therapeutic TRIM response in conditions like cancer (16).

Apart from the above described beneficial effects that can contribute to the TRIM against COVID-19 by normalization of blood glucose and lipid levels, since $\beta$-glucan can reduce the insulin resistance which has been proven in human studies (17), a therapeutic TRIM response proving beneficial in COVID-19 will occur when $\beta$-glucans are employed as a prophylactic agents and the state of immunological tolerance which exists in individuals with obesity, diabetes and dyslipidemia can perhaps be reversed by these $\beta$-glucans (18).

\section{CONCLUSION}

Therefore, in view of the excellent suggestion of TRIM induced by $\beta$-glucans as an "an efficient, low-cost and safe way" in a long term prophylactic setting applicable to different age groups of people and those with vacuous co-existing disease conditions in the defense against COVID-19 by Geller and Yan (1), we wish to reiterate that the metabolic effects of the $\beta$-glucans in terms of normalization of glucose levels, lipid levels and increase of insulin sensitivity significantly contributes to augment the TRIM response. Amidst the circumstances of a lack of definitive therapy or vaccine for COVID-19, Geller and Yan's timely suggestion of undertaking clinical trials to confirm the efficacy of TRIM response induced by $\beta$-glucans from various sources (1) needs immediate acknowledgement by the scientific community, while duly recognizing their contribution to COVID-19 defense through the above described potential metabolic pathways and their validation, for an efficient fight against this devastating pandemic.

\section{AUTHOR CONTRIBUTIONS}

$\mathrm{NI}$ and SA contributed to conception and design. SA drafted the manuscript. VD and MI performed critical revision of the manuscript. All authors contributed to the article and approved the submitted version. 


\section{REFERENCES}

1. Geller A, Yan J. Could the Induction of Trained Immunity by $\beta$-Glucan Serve as a Defense Against COVID-19? Front Immunol (2020) 11:1782. doi: 10.3389/fimmu.2020.01782

2. Rao KS, Suryaprakash V, Senthilkumar R, Preethy S, Katoh S, Ikewaki N, et al. Role of Immune Dysregulation in Increased Mortality Among a Specific Subset of COVID-19 Patients and Immune-Enhancement Strategies for Combatting Through Nutritional Supplements. Front Immunol (2020) 11:1548. doi: 10.3389/fimmu.2020.01548

3. Chen J, Raymond K. Beta-glucans in the treatment of diabetes and associated cardiovascular risks. Vasc Health Risk Manage (2008) 4(6):1265-72. doi: $10.2147 /$ vhrm.s3803

4. de Lucena T, da Silva Santos AF, de Lima BR, de Albuquerque Borborema ME, de Azevêdo Silva J. Mechanism of inflammatory response in associated comorbidities in COVID-19. Diabetes Metab Syndrome (2020) 14(4):597600. doi: 10.1016/j.dsx.2020.05.025

5. Singh VP, Bali A, Singh N, Jaggi AS. Advanced glycation end products and diabetic complications. Korean J Physiol Pharmacol: Off J Korean Physiol Soc Korean Soc Pharmacol (2014) 18(1):1-14. doi: 10.4196/kjpp.2014.18.1.1

6. Hussain A, Bhowmik B, do Vale Moreira NC. COVID-19 and diabetes: Knowledge in progress. Diabetes Res Clin Pract (2020) 162:108142. doi: 10.1016/j.diabres.2020.108142

7. Hariyanto TI, Kurniawan A. Dyslipidemia is associated with severe coronavirus disease 2019 (COVID-19) infection. Diabetes Metab Syndrome (2020) 14(5):1463-5. doi: 10.1016/j.dsx.2020.07.054

8. Ikewaki N, Rao KS, Archibold AD, Iwasaki M, Senthilkumar R, Preethy S, et al. Coagulopathy associated with COVID-19 - Perspectives \& Preventive strategies using a biological response modifier Glucan. Thromb J (2020) 18:27. doi: 10.1186/s12959-020-00239-6

9. Dedeepiya VD, Sivaraman G, Venkatesh AP, Preethy S, Abraham SJ. Potential effects of nichi glucan as a food supplement for diabetes mellitus and hyperlipidemia: preliminary findings from the study on three patients from India. Case Rep Med (2012) 2012:895370. doi: 10.1155/2012/895370

10. Yano H. Sophy Beta-Glucan is effective in alleviating increased blood sugar levels. In: Abstract presented at the 55th Conference of the Japanese Society of Nutrition and Dietetics (2008).

11. Ikewaki N, Iwasaki M, Abraham SJK. Biological response modifier glucan through balancing of blood glucose may have a prophylactic potential in COVID-19 patients. J Diabetes Metab Disord (2020) 19:1-4. doi: 10.1007/ s40200-020-00664-4
12. Wang S, Ma P, Zhang S, Song S, Wang Z, Ma Y, et al. Fasting blood glucose at admission is an independent predictor for 28-day mortality in patients with COVID-19 without previous diagnosis of diabetes: a multi-centre retrospective study. Diabetologia (2020) 63:2102-11. doi: 10.1007/s00125020-05209-1

13. Zhang B, Liu S, Zhang L, Dong Y, Zhang S. Admission fasting blood glucose predicts 30-day poor outcome in patients hospitalized for COVID-19 pneumonia. Diabetes Obes Metab (2020) 22:1955-57. doi: 10.1111/dom.14132

14. Ganesh JS, Rao YY, Ravikumar R, Jayakrishnan GA, Iwasaki M, Preethy S, et al. Beneficial effects of black yeast derived 1-3, 1-6 Beta Glucan-Nichi Glucan in a dyslipidemic individual of Indian origin-a case report. J Dietary Suppl (2014) 11(1):1-6. doi: 10.3109/19390211.2013.859211

15. Arts RJ, Joosten LA, Netea MG. Immunometabolic circuits in trained immunity. Semin Immunol (2016) 28(5):425-30. doi: 10.1016/ j.smim.2016.09.002

16. Ieronymaki E, Daskalaki MG, Lyroni K, Tsatsanis C. Insulin Signaling and Insulin Resistance Facilitate Trained Immunity in Macrophages Through Metabolic and Epigenetic Changes. Front Immunol (2019) 10:1330. doi: 10.3389/fimmu.2019.01330

17. Zheng J, Shen N, Wang S, Zhao G. Oat beta-glucan ameliorates insulin resistance in mice fed on high-fat and high-fructose diet. Food Nutr Res (2013) 57:22754. doi: 10.3402/fnr.v57i0.22754

18. Novakovic B, Habibi E, Wang SY, Arts R, Davar R, Megchelenbrink W, et al. $\beta$-Glucan Reverses the Epigenetic State of LPS-Induced Immunological Tolerance. Cell (2016) 167:1354-68.e14. doi: 10.1016/j.cell.2016.09.034

Conflict of Interest: SA is a shareholder in GN Corporation, Japan, which in turn is a shareholder in the manufacturing company of the AFO 202 Beta Glucan product and an applicant to several patents of relevance.

The remaining authors declare that the research was conducted in the absence of any commercial or financial relationships that could be construed as a potential conflict of interest.

Copyright (c) 2021 Ikewaki, Dedeepiya, Iwasaki and Abraham. This is an open-access article distributed under the terms of the Creative Commons Attribution License (CC BY). The use, distribution or reproduction in other forums is permitted, provided the original author(s) and the copyright owner(s) are credited and that the original publication in this journal is cited, in accordance with accepted academic practice. No use, distribution or reproduction is permitted which does not comply with these terms. 